\title{
Feasibility of HTS Magnet Option for Fusion Reactors ${ }^{*}$
}

\author{
Nagato YANAGI, Satoshi ITO ${ }^{1)}$, Yoshiro TERAZAKI ${ }^{2)}$, Kyohei NATSUME, Hitoshi TAMURA, \\ Shinji HAMAGUCHI, Toshiyuki MITO, Hidetoshi HASHIZUME ${ }^{1)}$, Junji MORIKAWA ${ }^{3)}$, \\ Yuichi OGAWA ${ }^{3)}$, Masataka IWAKUMA ${ }^{4)}$ and Akio SAGARA \\ National Institute for Fusion Science, 322-6 Oroshi-cho, Toki, Gifu 509-5292, Japan \\ ${ }^{1)}$ Department of Quantum Science and Energy Engineering, Tohoku University, 6-6-01-2 Aoba, Aramaki, Aoba-ku, Sendai, \\ Miyagi 980-8579, Japan \\ ${ }^{2)}$ The Graduate University for Advanced Studies, 322-6 Oroshi-cho, Toki, Gifu 509-5292, Japan \\ ${ }^{3)}$ Graduate School of Frontier Sciences, The University of Tokyo, Kashiwa, Chiba 277-8568, Japan \\ ${ }^{4)}$ Research Institute of Superconductor Science and Systems, Kyushu University, 744 Motooka, Nishi-ku, Fukuoka \\ 819-0395, Japan
}

(Received 27 April 2013 / Accepted 22 December 2013)

\begin{abstract}
Conceptual design studies are being carried out on the application of high-temperature superconducting (HTS) conductors and coils to the magnet systems of fusion reactors. A 100-kA-class HTS conductor is required to be applied at high magnetic fields of $>12 \mathrm{~T}$. A simple stack of YBCO tapes embedded in copper and stainless-steel jackets is found to be a practical approach to producing large-scale conductors that exhibit high cryogenic stability and mechanical rigidity. The feasibility of the segmented fabrication method for large complex HTS coils, such as the helical coils in the LHD-type helical fusion reactor FFHR-d1, is being investigated by developing mechanical bridge-type lap joint technology of HTS conductors.
\end{abstract}

(C) 2014 The Japan Society of Plasma Science and Nuclear Fusion Research

Keywords: high-temperature superconductor (HTS), YBCO, fusion reactor, helical, FFHR-d1

DOI: $10.1585 /$ pfr.9.1405013

\section{Introduction}

Owing to the significant progress that has been made in wire production technology in recent years, copperoxide based high-temperature superconducting (HTS) materials are now being applied to a number of prototype electrical devices, such as power cables, transformers, energy storage systems and motors [1]. The production of largescale HTS magnets for use in fusion reactors has also been considered [2,3]. Though it is still widely believed that HTS materials are fragile, it is actually possible to fabricate strong coils using rare-earth barium copper oxide (REBCO) tape. In this type of tape, a substrate composed of a Ni alloy such as Hastelloy, with a high yield strength of $>1 \mathrm{GPa}$, is coated with a REBCO material such as YBCO (where Y represents yttrium) [1].

The application of HTS materials in fusion research began with the floating coil of the Mini-RT device which has been operational since 2003 at the University of Tokyo [4]. In this coil, Ag-sheathed Bi-2223 tape was used, which was the only available HTS at that time. The larger RT-1 device was built in 2006 using improved Bi2223 tape, and stable levitation for more than 6 hours was achieved [5,6]. Recently, the floating coil of the Mini-RT was upgraded using the latest GdBCO tape for better per-

author'se-mail: yanagi@LHD.nifs.ac.jp

*) This article is based on the invited talk at the 29th JSPF Annual Meeting (2012, Fukuoka) formances $[7,8]$.

As is well known, the biggest advantage of HTS materials is their high critical current density up to high magnetic fields of $>30 \mathrm{~T}$. Another important advantage is their high cryogenic stability (low quench probability) due to the increased heat capacity of the conductor composites at temperatures $>20 \mathrm{~K}$. This is in remarkable contrast to the situation for $\mathrm{Nb}$-based low-temperature superconducting (LTS) materials, which relies on the heat capacity of helium at $\sim 4 \mathrm{~K}$. Figure 1 shows the temperature dependence of the volumetric specific heat $C_{p} \rho\left(C_{p}\right.$ is the spe-

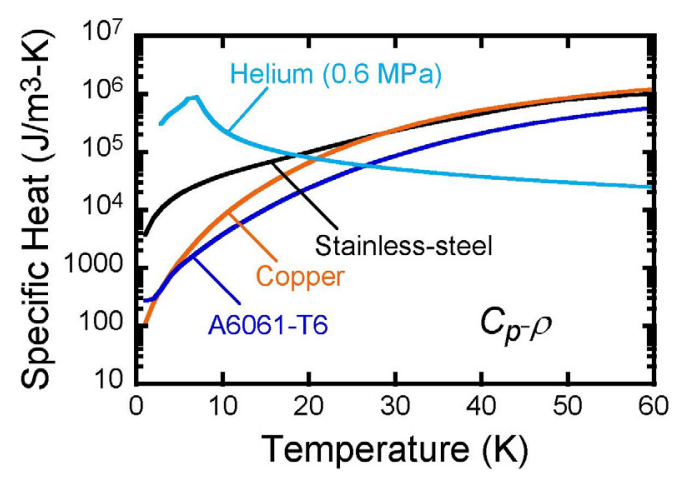

Fig. 1 Volumetric specific heat for metals and helium (pressure: $0.6 \mathrm{MPa}$ ) as a function of temperature. 
cific heat and $\rho$ is the density) for several metals and for helium. As can be seen, the $C_{p} \rho$ for stainless-steel and copper at $30 \mathrm{~K}$ are equivalent as that for helium at $4 \mathrm{~K}$. The stability margin (allowable disturbance per unit volume) for a conductor can be estimated using $w=C_{p} \rho \Delta T$, where $\Delta T$ is the temperature margin). Considering that $\Delta T$ could be as much as $\sim 10 \mathrm{~K}$ for HTS conductors (unlike $1-2 \mathrm{~K}$ for LTS), $w$ is found to be $\sim 2 \mathrm{MJ} / \mathrm{m}^{3}$ ( $2 \mathrm{~J} / \mathrm{cc}$ ) for a temperature change of $20-30 \mathrm{~K}$ under adiabatic conditions. This is even larger than that measured for the presently used cablein-conduit (CIC) conductors that are internally cooled by forced flow of supercritical helium at $\sim 4.5 \mathrm{~K}$ [9]. The stability margin allows a heat generation $w^{\prime}=B j \delta$ due to the displacement $\delta$ of the conductor caused by an electromagnetic force associated with magnetic field $B$ and current density $j$. For $B=12 \mathrm{~T}$ and $j=25 \mathrm{~A} / \mathrm{mm}^{2}$, a stability margin of $2 \mathrm{MJ} / \mathrm{m}^{3}$ allows a disturbance (winding motion) of $\delta \sim 6 \mathrm{~mm}$, which is unlikely to occur in a real magnet.

Considering these advantages, the conceptual design studies on the use of HTS materials in tokamak reactors were carried out for ARIES-AT [10] and VECTOR [11] more than 10 years ago. Along with the design of VECTOR, a 10-kA-class CIC conductor using round Bi-2212 HTS strands was successfully tested [12]. Although, these studies and conductor development have since been suspended, the HTS option is now being considered for the helical fusion reactor, and conductor development has been underway since 2005 .

The present paper considers several aspects of HTS application to the magnet coils of fusion reactors, taking the LHD-type helical reactor FFHR-d1 as an example.

\section{Design of a 100-kA-Class HTS Con- ductor for DC Magnets of Fusion Reactors}

As an example of the application of HTS conductors to the magnets of fusion reactors, the conceptual design of the LHD-type helical fusion reactor is considered. The present design, FFHR-d1, is equipped with a pair of continuously wound helical coils, having a major radius of $15.6 \mathrm{~m}$, a minor radius of $3.744 \mathrm{~m}$ and a helical pitch number of 10 , that produce a $4.7 \mathrm{~T}$ toroidal magnetic field to generate $3 \mathrm{GW}$ of fusion power [13]. The stored magnetic energy is $160 \mathrm{GJ}$ and a $100-\mathrm{kA}$ conductor is required to be used at the maximum magnetic field of $\sim 12 \mathrm{~T}$. The primary selection for the helical windings is a CIC conductor using $\mathrm{Nb}_{3} \mathrm{Al}$ strands [14], which is regarded as an extension of the ITER technology. However, considering the difficulties related to CIC conductors, such as the complicated plumbing required and the degradation of the critical current due to strain, an indirectly-cooled LTS conductor using $\mathrm{Nb}_{3} \mathrm{Sn}$ strands and an aluminum-alloy jacket has also been considered [15]. The other option for indirect cooling is to use a HTS conductor.

To fabricate a high-current-capacity HTS conductor, the general approach has been to employ the same concepts that have been widely applied to LTS conductors. In order to make the conductor resistant to magnetic instabilities (flux jumps), and to achieve high cryogenic stability by avoiding a non-uniform current distribution, round multi-filamentary wires containing thin filaments should be used as strands, and the strands should be twisted and transposed. To achieve this, several methods for producing HTS stranded cable have been developed, such as the "Roebel-assembled" cable [16], "twist-stacked" cable [17] and "Conductor On Round Core (CORC)" cable [18]. However, since these methods are complex and expensive, we propose a simple stacking of HTS tapes. This should be particularly effective for DC magnets by allowing a formation of non-uniform current distribution, since a HTS has a high cryogenic stability. Using this stacking approach, a mechanically strong conductor can be fabricated at low cost.

Figure 2 shows an example of the 100-kA-class conductor design for FFHR-d1. Forty YBCO tapes, each with a width of $15 \mathrm{~mm}$, are simply stacked within a copper jacket. The operation temperature is $20 \mathrm{~K}$. The conductor has a round outer shape produced by a stainlesssteel jacket. The major specifications for the conductor are listed in Table 1. We should point out that this is actually an older design and has since been changed [19]. The most recent conductor design has internal insulation, and use a stainless-steel jacket with a square outer shape, which is directly cooled by gas helium. However, it is also worthwhile to evaluate the conductor design shown in Fig. 2, not only with regard to FFHR-d1, but also other applications such as the toroidal field (TF) coils of tokamak reactors.

Simple stacking of HTS tapes may also be applicable to TF coils provided the flux changes by vertical field coils are slow in tokamak reactors. It should be noted that in previous work on the VECTOR design, simple stacking of YBCO tapes was considered. If the field changes are too fast, it is better to reduce both AC losses and the occurrence of non-uniform current distribution, which may be possible by transposing the HTS tapes [20]. This can be

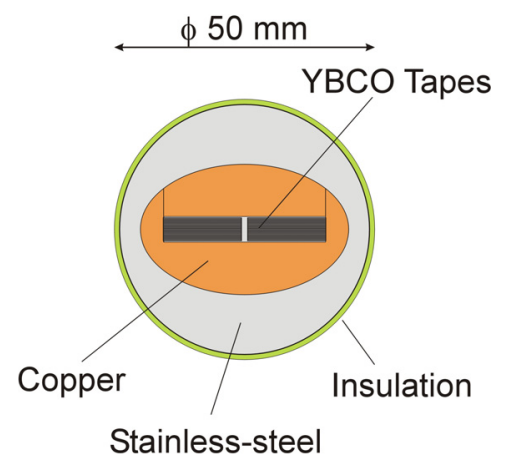

Fig. 2 Cross-sectional illustration of a 100-kA-class HTS conductor design for FFHR-d1 helical coils. 
Table 1 Major specifications of a 100-kA-class HTS conductor design for FFHR-d1.

\begin{tabular}{|l|c|}
\hline Operation current (12 T, 20 K) & $94 \mathrm{kA}$ \\
\hline Conductor size & $\phi 50 \mathrm{~mm}$ \\
\hline Current density & $40 \mathrm{~A} / \mathrm{mm}^{2}$ \\
\hline Superconducting material & YBCO \\
\hline Width of YBCO tape & $15 \mathrm{~mm}$ \\
\hline Thickness of YBCO tape & $0.22 \mathrm{~mm}$ \\
\hline $\begin{array}{c}\text { Critical current of a } 15 \mathrm{~mm} \\
\text { width tape (77 K, self-field) }\end{array}$ & $>900 \mathrm{~A}$ \\
\hline Number of HTS tapes & 40 \\
\hline Configuration of HTS tapes & Simple-stacking \\
\hline Jacket material & Stainless-steel \\
\hline Stabilizer & Oxygen-free copper \\
\hline Cooling method & Indirect cooling \\
\hline
\end{tabular}

achieved using a Roebel-assembled conductor. To more easily produce Roebel conductors, the Roebel-MITO (Meandering for Inter-Transposition Optimization) method has been proposed, and this ensures an on-demand realization of transposition using soldered joints [21]. A similar idea was considered for the YBCO conductors of VECTOR [22].

\section{Design of HTS Coil Winding Pack}

Figure 3 shows a schematic cross-section of the winding pack of the FFHR-d1 helical coil using the type of HTS conductor seen in Fig. 2. The total current in the helical coil is $36.66 \mathrm{MA}$ and the current density in the winding region is $24.45 \mathrm{~A} / \mathrm{mm}^{2}$, which is larger than that for the ITER TF coils $\left(20 \mathrm{~A} / \mathrm{mm}^{2}\right)$. The winding pack consists of 390 turns of HTS conductors, each with an operation current of $94 \mathrm{kA}$, installed in a stainless-steel "internal plate" with circular grooves. This is the same concept employed for the CIC conductor version of the FFHR helical coils [14]. The internal plate is the layer winding version of the "radial plate" used in the ITER TF coils with pancake winding. In Fig. 3, the internal plate is cooled by gas helium flowing through cooling pipes imbedded within it. The conductors are cooled indirectly by the internal plate through thermal conduction. To enhance the cooling capabilities of the internal plate, innovations such as the use of oscillating heatpipes should be considered [23]. Once again, we note that the winding pack shown in Fig. 3 is our former design, and a different configuration is employed associated with the more recent square-type conductor design having internal insulation cooled directly by gas helium [19].

As shown in Fig. 3, the orientation of YBCO tapes is adjusted so that the plane of each tape is parallel to the magnetic field. This arrangement is used in order to mitigate the dependence of the critical current on the magnetic field orientation, which is still a remaining issue with RE$\mathrm{BCO}$ tape. This alignment also dramatically reduces $\mathrm{AC}$ losses [24]. This is ananalogous to the reduction in AC

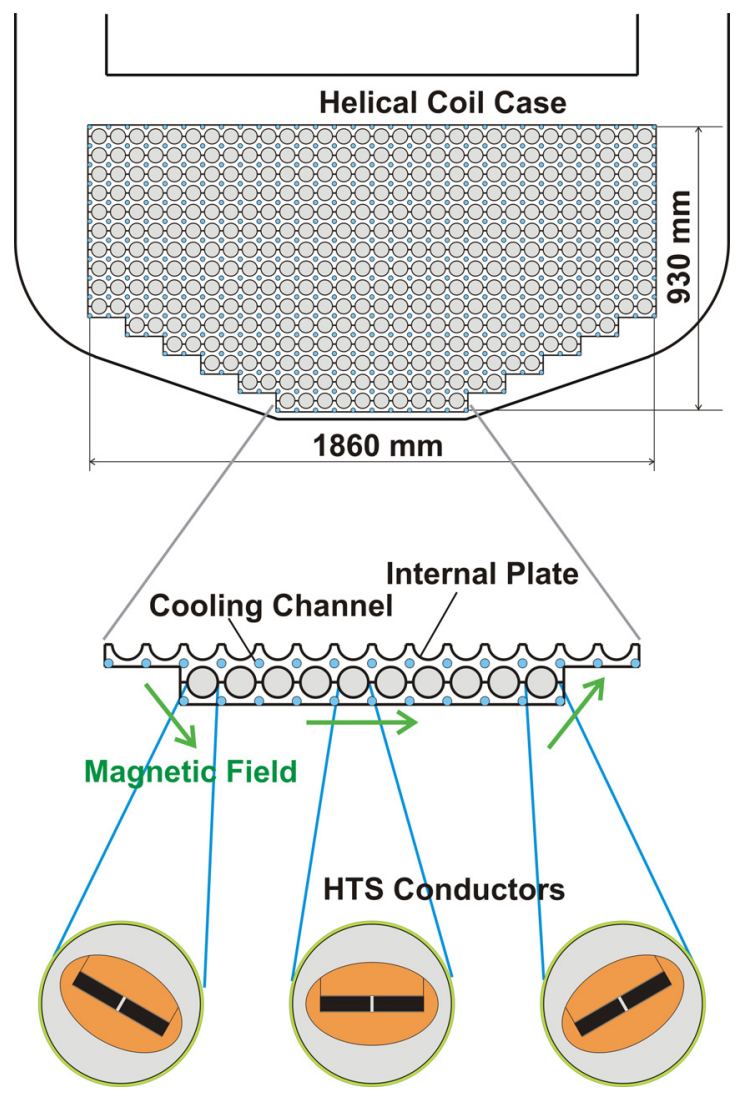

Fig. 3 Schematic cross-section of helical coil windings using indirectly-cooled HTS conductors for FFHR-d1.

losses that occurred when a flat Rutherford-type cable with $\mathrm{NbTi} / \mathrm{Cu}$ strands was wound by twisting the cable being parallel to the magnetic field [25]. The arrangement shown in Fig. 3 also reduces the production of shielding currents, and therefore the error magnetic field [26].

When $3 \mathrm{GW}$ of fusion power is being generated by FFHR-d1, it is required to remove $\sim 500 \mathrm{~W} / \mathrm{m}^{3}$ of nuclear heating in the winding pack at the inboard side of the torus ( $\sim 100 \mathrm{~W} / \mathrm{m}^{3}$ at the outboard side) [13]. A one-dimensional heat conduction analysis using the thermal conductivities of conductor composites such as stainless-steel, copper and epoxy (insulation), shows that a temperature difference of only $1 \mathrm{~K}$ develops between the HTS tape and the internal plate.

Over a full 30 years of operation, the neutron fluence and the gamma-ray dose are expected to be $\sim 1 \times 10^{23} / \mathrm{m}^{2}$ and $\sim 100 \mathrm{MGy}$, respectively, near the innermost layer of the helical coils at the inboard side of the torus. These values are both marginal for superconductors and insulators [27]. We are also exploring the possibility of using inorganic materials such as ceramics as insulators. Furthermore, optimization of the coil configuration is underway in order to increase the amount of space at the inboard side of the torus, so that the shielding can be increased by $\sim 100 \mathrm{~mm}$, leading to a reduction in the neutron and gamma ray flux by about 10 times. 


\section{Segmented Fabrication of Large Complex Coils using HTS Conduc- tors}

To facilitate the winding of large complex coils, such as the helical coils of FFHR-d1, one possibility is segmented fabrication using HTS conductors [26, 28-31] instead of continuous winding using LTS conductors. Segmented fabrication was first proposed for helical reactors in the 1980's with the so-called "demountable" concept [28], which is illustrated in Fig. 4 (a). A similar concept was proposed in 2001 using a HTS approach that took advantage of the reduced refrigeration power required due to the higher temperature operation [29]. However, with present-day technology, it is difficult to find a realistic solution to forming simultaneous connections between hundreds of turns of winding conductors. Thus, we proposed the idea of connecting "conductor segments" as shown in Fig. 4 (b), instead of "coil segments" [26]. For the bridgetype conductor joint shown in Fig. 5 (a), a mechanical joint technique developed at Tohoku University can be applied instead of the formerly considered soldering method [32]. For a mechanical lap joint, the joint resistivity is estimated to be $\sim 10 \mathrm{p} \Omega \mathrm{m}^{2}[33,34]$, giving an overall joint resistance of $\sim 1 \mathrm{n} \Omega$ for a 100-kA conductor (consisting of 2 connections per joint with 40 HTS tapes, each having a $50-\mathrm{mm}$ joint length). The entire helical coil system has 7,800 joints (390 turns, 10 segments, 2 coils), and requires a $\sim 5 \mathrm{MW}$ increase in the electrical power to the cryoplant for a coil operation temperature of $20 \mathrm{~K}$. This is acceptable given the power requirements of $\sim 30 \mathrm{MW}$ for a LTS system operated at $4 \mathrm{~K}$. To further reduce the refrigeration power, we propose the "finger-type" joint shown in Fig. 5 (b), which has a 10 times larger contact area and requires only $500 \mathrm{~kW}$ of electricity for the cryoplant.

A rough outline of the in-situ construction procedure for the helical coils is as follows:

1. The helical coil cases are assembled by connecting half-helical-pitch sections along the torus to form the helical coil structure.

2. To prepare the HTS conductors, the stainless-steel and copper jackets of the conductor are pre-bent into a helical shape with each $\sim 16 \mathrm{~m}$ length in a sufficient tolerance. YBCO tapes are then embedded into the copper jacket (applying limited strain) and the stainless-steel jacket is welded along the conductor. The insulator should be attached to the outer surface of the stainless-steel jacket at this stage.

3. One by one, the half-helical-pitch conductors are placed into the circular grooves in the internal plate of the helical coil case.

4. Joints are then formed between two conductors as described above. To ensure the mechanical strength of the joints, welding of the stainless-steel jacket is inevitable.

5. After each joint is formed, an inspection should be (a)

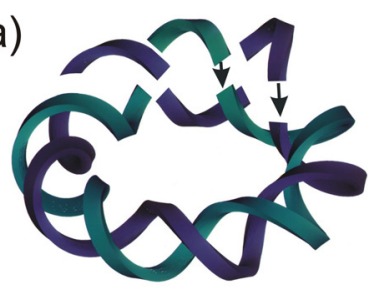

(b)

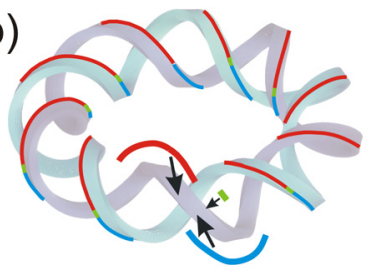

(c)

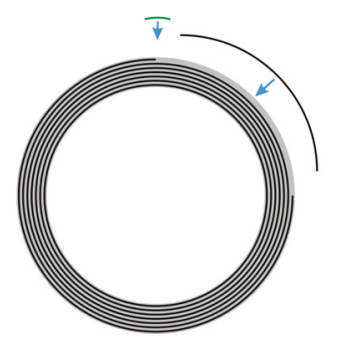

Fig. 4 Conceptual illustrations of the segment-fabrication method for (a) helical coils with coil segments, (b) helical coils with conductor segments and (c) vertical field coils with conductor segments.

(a)

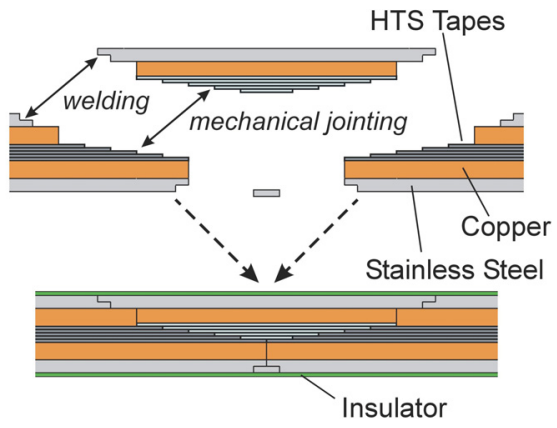

(b)

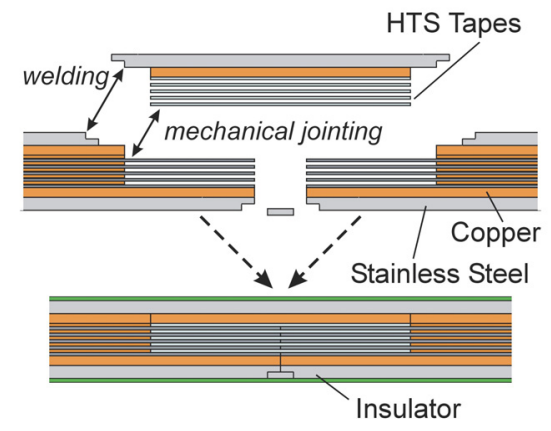

Fig. 5 Schematic illustrations of HTS conductor joints: (a) "bridge-type" lap joint and (b) "finger-type" lap joint.

carried out using a technique such as ultrasound or Xrays. Mechanical testing can also be performed. For quality assurance, the joint formation and inspection 
should be done automatically using industrial robots (not by hand). In the inspection stage, the electrical resistance of the joint can be measured while cooling a joint section with liquid nitrogen, should it be required.

6. Insulation should be separately attached around a joint after the mechanical jointing process is finished.

7. Using the above procedure, one layer of windings is formed, and the internal plate for the next layer (fabricated in pieces and welded together along the torus on site) is then placed onto the lower layer. In this way, all the layers (fifteen in the example of Fig. 3) are fabricated using 7,800 half-helical-pitch conductors connected one by one to form the continuously wound helical coils.

8. After the winding process is completed, the top lids of the helical coil cases are attached and welded.

9. In order to fill the gaps within the winding area, vacuum pressure impregnation (VPI) should ideally be carried out at a temperature of $\sim 150$ centigrade.

Regarding the final procedure, since performing VPI for such a large coil is expected to be difficult, we have proposed an alternative to the VPI process [19]. Nevertheless, the use of VPI is worth exploring further.

Segment fabrication could also be applied to the vertical field coils, as shown in Fig. 4 (c). On the other hand, demountable coils could be a future option for the manufacture of commercial helical-type reactors, and also for producing medium-scale TF coils for tokamak reactors that allow blanket maintenance [35].

\section{Development of 100-kA-Class HTS Conductor and Joint for FFHR-d1}

Large-scale HTS conductor development for FFHR began with a 10-kA-class conductor in 2005 [36, 37]. In 2012, a 30-kA-class conductor sample was fabricated using the latest GdBCO tape produced by Fujikura Ltd. Twenty tapes were simply stacked in 10 layers and 2 rows [19]. In the sample, a bridge-type mechanical lap joint developed at Tohoku University $[33,34,38]$ was used so that the racetrack shaped sample formed a short circuit. A sample current was induced by changing the bias magnetic field, and a critical current of $45 \mathrm{kA}$ was measured at $20 \mathrm{~K}$ and $6 \mathrm{~T}$ [39]. Stable operation at a current of $>30 \mathrm{kA}$ was sustained for more than $20 \mathrm{~min}$ at $30 \mathrm{~K}$. The maximum current was $70 \mathrm{kA}$ at $4 \mathrm{~K}$ and $1 \mathrm{~T}$ with a fast ramping rate of $1 \mathrm{kA} / \mathrm{s}$. No premature quench was observed, which confirms the high cryogenic stability of the HTS conductor. Details concerning this conductor test are described elsewhere [19,38,39].

\section{Summary}

The application of HTS conductors to fusion reactor magnets seems feasible owing both to the rapid progress in wire production technology and development of highcurrent capacity conductors at National Institute for Fusion Science. Simple stacking of REBCO coated-conductor tapes is a promising method for producing cryogenically stable and mechanically rigid conductors for DC magnets at a reasonable cost. Segmented fabrication of large complex coils, such as the helical coils for the LHD-type helical reactor FFHR-d1, is expected to be possible using mechanical bridge-type lap joints.

\section{Acknowledgments}

The authors are grateful to S. Imagawa, J. Miyazawa, T. Goto, T. Tanaka and the members in the Fusion Engineering Research Project at NIFS for valuable discussions and encouragement. One of the authors (N. Y.) thanks G. Bansal, T. Hemmi, R. Champailler and H. Noguchi who contributed to starting 10-kA-class HTS conductor development and performed feasibility studies of the HTS magnet option for FFHR.

[1] Y. Shiohara et al., Physica C 468, 1498 (2008).

[2] W.H. Fietz et al., Fusion Eng. Des. 75, 105 (2005).

[3] P. Komarek, Fusion Eng. Des. 81, 2287 (2006).

[4] Y. Ogawa et al., J. Plasma Fusion Res. 79, 643 (2003).

[5] S. Mizumaki et al., IEEE Trans. Appl. Supercond. 16, 918 (2006).

[6] Y. Ogawa et al., Plasma Fusion Res. 4, 020 (2009).

[7] Y. Ogawa et al., to be published in Plasma Fusion Res.

[8] K. Natsume et al., IEEE Trans. Appl. Supercond. 24, 4601104 (2014).

[9] N. Koizumi et al., Nucl. Fusion 45, 431 (2005).

[10] F. Dahlgren et al., Fusion Eng. Des. 167, 139 (2006).

[11] T. Ando, S. Nishio and H. Yoshimura, IEEE Trans. Appl. Supercond. 14, 1481 (2004).

[12] T. Isono et al., IEEE Trans. Appl. Supercond. 13, 1512 (2003).

[13] A. Sagara et al., Fusion Eng. Des. 87, 594 (2012).

[14] S. Imagawa et al., Nucl. Fusion 49, 075017 (2009).

[15] K. Takahata et al., Fusion Eng. Des. 82, 1487 (2007).

[16] W. Goldacker et al., Supercond. Sci. Technol. 22, 034003 (2009).

[17] M. Takayasu, Supercond. Sci. Technol. 25, 014011 (2012).

[18] D.C. van der Laan, Supercond. Sci. Technol. 22, 065013 (2009).

[19] N. Yanagi et al., IEEE Trans. Appl. Supercond. 24, 4202805 (2014).

[20] M. Iwakuma et al., Supercond. Sci. Technol. 23, 075009 (2010).

[21] N. Yanagi et al., Physics Procedia 27, 444 (2012).

[22] T. Ando, S. Nishio, SOFE05 Knoxville, TN, USA (2005).

[23] T. Mito et al, IEEE Trans. Appl. Supercond. 21, 2470 (2011).

[24] M. Iwakuma et al., Physica C 469, 1726 (2009).

[25] T. Mito et al., IEEE Trans. Appl. Supercond. 17, 1973 (2007).

[26] N. Yanagi et al., Fusion Sci. Technol. 60, 648 (2011).

[27] L.A. El-Guebaly and The ARIES Team, Fusion Eng. Des. 38, 139 (1997).

[28] K. Uo et al., Proc. 14th SOFT, 1727 (1986).

[29] H. Hashizume et al., Fusion Eng. Des. 63, 449 (2002). 
[30] G. Bansal et al., Plasma Fusion Res. 3, S1049 (2008).

[31] N. Yanagi et al., Plasma Fusion Res. 5, S1026 (2010).

[32] Y. Terazaki et al., Plasma Fusion Res. 7, 2405027 (2012).

[33] S. Ito et al., IEEE Trans. Appl. Supercond. 22, 6400204 (2012).

[34] K. Kawai et al., IEEE Trans. Appl. Supercond. 23, 4801704 (2013).

[35] L. Bromberg et al., Fusion Sci. Technol. 60, 635 (2011).
[36] G. Bansal et al., IEEE Trans. Appl. Supercond. 18, 1151 (2008).

[37] R. Champailler et al., IEEE Trans. Appl. Supercond. 20, 1565 (2010).

[38] S. Ito et al., IEEE Trans. Appl. Supercond. 24, 4602305 (2014).

[39] Y. Terazaki et al., IEEE Trans. Appl. Supercond. 24, 4801305 (2014). 